\begin{tabular}{|c|l|}
\hline Title & $\begin{array}{l}\text { Dynamics and structure of hydrogen-bonding glass formers: Comparison between hexanetriol and sugar al cohols based } \\
\text { on dielectric relaxation }\end{array}$ \\
\hline Author(s) & Nakanishi, Masahiro; Nozaki, Ryusuke \\
\hline Citation & $\begin{array}{l}\text { Physical review E, 81(4), 041501 } \\
\text { https://doi.org/L0.1103/PhySRevE.81.041501 }\end{array}$ \\
\hline Issue Date & 2010-04 \\
\hline Doc URL & http://hdl.handle.net/2115/43061 \\
\hline Rights & ○2010 The A merican Physical Society \\
\hline Type & article \\
\hline File Information & PRE81-4_041501.pdf \\
\hline
\end{tabular}

Instructions for use 


\title{
Dynamics and structure of hydrogen-bonding glass formers: Comparison between hexanetriol and sugar alcohols based on dielectric relaxation
}

\author{
Masahiro Nakanishi (中西真大) and Ryusuke Nozaki（野齐龍介) \\ Department of Physics, Faculty of Science, Hokkaido University, Sapporo 060-0810, Japan
}

(Received 10 December 2009; published 8 April 2010)

\begin{abstract}
Broadband dielectric spectra of supercooled 1,2,6-hexanetriol are presented in order to reveal physical picture behind a glass transition of polyhydric alcohols. It has been reported so far that temperature dependences of $\alpha$ relaxation time for sugar alcohols exhibit systematic trend against number of carbon atoms or $\mathrm{OH}$ groups per molecule. However, because each molecule is composed of equal number of carbon atoms and $\mathrm{OH}$ groups in the case of the reported sugar alcohols, the more dominant parameter to govern the $\alpha$ relaxation dynamics has not been discussed. By using a chemical structure of the hexanetriol composed of the deferent number of carbon and $\mathrm{OH}$, it is possible to determine the dominant parameter. From temperature dependence of $\alpha$ relaxation times, it is strongly supported that the number of $\mathrm{OH}$ groups is the dominant parameter. Furthermore, from an analysis of static dielectric constant, it is suggested that local hydrogen-bonding structure is similar among all polyhydric alcohols. From these two results, a simple picture of the origin of the systematic character is proposed.
\end{abstract}

DOI: 10.1103/PhysRevE.81.041501

PACS number(s): 64.70.pm, 77.22.Gm, 61.25.Em

\section{INTRODUCTION}

A variety of materials, such as colloidal systems, polymers, molten salts, metals, molecular liquids, hydrogenbonding liquids and so on, exhibits glass transition. Among these glass-forming liquids, a special attention has been paid on the hydrogen-bonding liquids because of its peculiar features, such as anomalous relation between fragility index and specific-heat change at the glass transition [1]. Polyhydric alcohol is one of the hydrogen-bonding liquids, where each molecule has two or more $\mathrm{OH}$ groups. In general, polyhydric alcohols exhibit the glass transition at temperatures $\left(T_{g}\right)$ relatively higher than those of usual molecular liquids with similar molecular size. Sugar alcohols being considered in this work are one of the polyhydric alcohols, which consist of a linear backbone chain with $\mathrm{OH}$ groups attached to every carbon atoms (see Fig. 1). Glycerol is a typical glass-forming polyhydric alcohol and a lot of studies have been performed by various experimental techniques and its dynamics has been investigated from a variety of aspects so far [2-9]. In the study on dynamics of hydrogen-bonding liquid, dielectric spectroscopy is one of the powerful techniques because the $\mathrm{OH}$ groups mainly characterizing the liquid are dielectrically active.

Recently, a systematic change in the dielectric spectrum of sugar alcohols against the backbone chain length has been reported [10-13]. In these studies, sugar alcohols involving sorbitol, xylitol, threitol and glycerol with 6, 5, 4, 3 distinct number of carbon atoms $\left(N_{C}\right)$ are examined. Figure 2 shows the dielectric spectrum [14] of these materials at few degrees above $T_{g}[12,13]$. In general, two relaxation processes, main dispersion ( $\alpha$ relaxation) and broad secondary dispersion $(\beta$ relaxation) are observed in sugar alcohols. With increasing $N_{C}$, dielectric relaxation intensity of the $\alpha$ relaxation decreases whereas the $\beta$ relaxation develops into an apparent shoulder from the excess wing. From the viewpoint of temperature dependence of their relaxation times, the $\alpha$ relaxation follows the Vogel-Fulcher-Tammann (VFT) equation
[15-17] while the $\beta$ relaxation keeps the Arrhenius manner. For the $\alpha$ relaxation, a systematic increase of $T_{g}$ and fragility index [1] with increasing $N_{C}$ has been reported. On the other hand, the $\beta$ relaxation exhibits common temperature dependence for all the sugar alcohols.

These characteristic features imply that a common mechanism dominates the glass transition of these materials. The question now is to determine the most dominant parameter to describe the systematic feature of the $\alpha$ process. Such question must be significant to construct a reduced model for these materials.

Previously, several theories and concepts on glass transition have been proposed, for instance, free-volume theory $[18,19]$, Adam-Gibbs theory [20], mode-coupling theory [21], coupling model [22,23], energy landscape [24], and so on. To consider the possible candidate for the dominant parameter, it would be natural to start the discussion with generally accepted classical theories, free-volume theory and Adam-Gibbs theory, because these theories are based on simple concepts. The former considers the size of the molecule while the latter describes the cooperative activation energy. In the case of sugar alcohols, it can be considered that the former is clearly related to the length of the carbon chain, namely, $N_{C}$, whereas the latter is associated with the structure of hydrogen bonding. For the hydrogen-bonding liquids including the sugar alcohols, an existence of hydrogen-bonding network has been reported from several experiments and simulations [25-29]. In this network, some $\mathrm{OH}$ groups connect to the other $\mathrm{OH}$ groups and therefore reorientational dynamics of $\mathrm{OH}$ group is strongly associated with each other. In this case, the number of $\mathrm{OH}$ groups per molecule $\left(N_{O H}\right)$ or the density of $\mathrm{OH}$ groups is considered to play an important role on their systematic behavior. In the sugar alcohol system, $N_{O H}$ is always equal to $N_{C}$ and therefore $\mathrm{OH}$ group density is virtually constant. Consequently, it is concluded that $\mathrm{OH}$ group density is not the origin of the systematic character.

From the above considerations, the parameters $N_{C}$ and/or $N_{O H}$ are considered to be important to understand the glass 


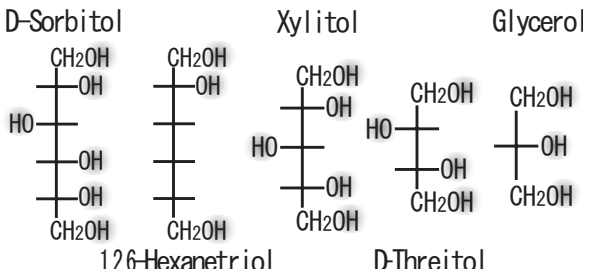

FIG. 1. Structures of the molecules involved in this paper. D-sorbitol, 1,2,6-hexanetriol, xylitol, D-threitol, glycerol are depicted as Fischer projection (presented data for hexanetriol and threitol are based on the mixture of $\mathrm{L}$ and $\mathrm{D}$ ). These sugar alcohols are molecule with the same number of $\mathrm{OH}$ group as that of carbon. The number of carbon atoms of hexanetriol is equal to that of sorbitol, whereas the number of $\mathrm{OH}$ groups is same as that of glycerol.

transition. However, because $N_{C}$ is always equal to $N_{O H}$ for sugar alcohols, the difference of their effects on the $\alpha$ relaxation cannot be distinguished. In order to solve this problem, we examine the 1,2,6-hexanetriol $\left(N_{C}=6, N_{O H}=3\right)$ (Fig. 1) with a broadband dielectric spectroscopy. In this material, $N_{C}$ is equal to that of sorbitol $\left(N_{C}=6, N_{O H}=6\right)$ and $N_{O H}$ is equal to that of glycerol $\left(N_{C}=3, N_{O H}=3\right)$. By comparing these glass transition behaviors, the most dominant parameter can be determined and a more detailed understanding on the systematic character in sugar alcohols should be attained.

In the present paper, we discuss the broadband dielectric spectra and its temperature dependence especially regarding $\alpha$ relaxation. Moreover, analysis of static dielectric constant and consequently derived Kirkwood $g$ factor are presented.

\section{MATERIALS AND METHODS}

Liquid 1,2,6-hexanetriol was purchased from Merck $\mathrm{KGaA}$. To remove the water, the sample was kept in a vacuum chamber at $373 \mathrm{~K}$ for more than $10 \mathrm{~h}$.

Dielectric measurements were carried out from $1 \mu \mathrm{Hz}$ to $10 \mathrm{GHz}$. To cover the wide frequency range, five different measuring systems were used. From $1 \mu \mathrm{Hz}$ to $100 \mathrm{mHz}$, self-made time domain spectrometer [30,31] was employed, which consists of electrometer (Keithley 6512), oscilloscope (Hewlett-Packard 54600B) and D/A converter (MCI engineering DAM-702GPC). A sample cell $A$ with guarding electrode was employed with a capacitance of about $45 \mathrm{pF}$. A quasistep voltage produced by D/A converter was applied between electrodes and the subsequent response current, which consists of fast and slow components, was amplified by electrometer. The fast-decaying current was measured by oscilloscope through the electrometer preamp output. On the other hand, the slow-decaying current was recorded by personal computer through repeated reading of electrometer indicator. Each of the voltage and the current was Fourier transformed into frequency domain and complex permittivity was calculated. It should be noted that, before the measurement, the sample to be measured was equilibrated at the measurement temperature. From $10 \mathrm{mHz}$ to $1 \mathrm{kHz}$, an ac phase analysis [30] was employed using a current amplifier (Keithley 428), digital sampling oscilloscope (Tektronix TDS210) and function generator (Yokogawa FG110). Cell $A$

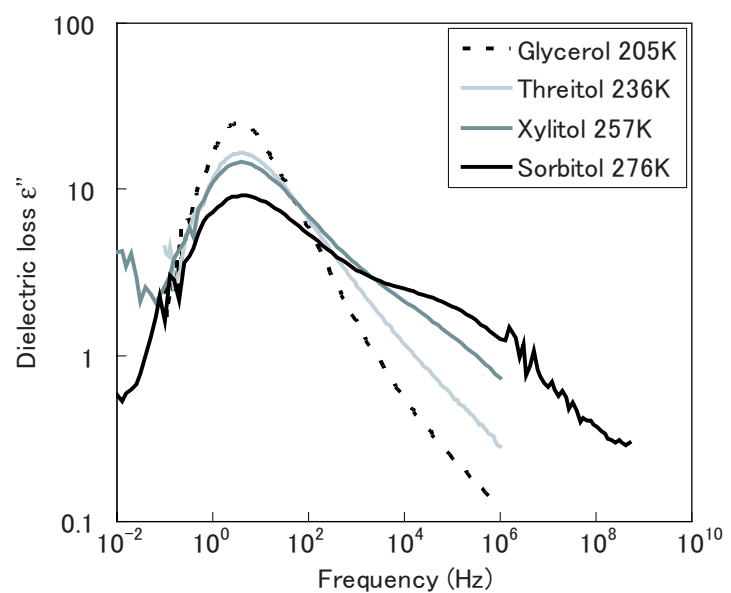

FIG. 2. (Color online) Previously reported systematic variation in dielectric loss spectra of sugar alcohols [12,13]. Dielectric loss, $\varepsilon^{\prime \prime}$, is plotted against frequency. Each curve is obtained at different temperature where the peak of main dispersions is on the unique frequency. Intensity of main dispersions increases with decreasing chain length. On the other hand, that of secondary dispersions decreases with chain length. Glass transition temperature decreases with chain length.

was also used in this system. From $40 \mathrm{~Hz}$ to $110 \mathrm{MHz}$, an impedance analyzer (Agilent 4294A) was employed as a two-terminal coaxial measuring system with a newly developed dielectric cell $B$. Using cell $B$ which has a circular parallel-plate capacitor connected to the coaxial port via coaxial coupling, we have successfully cover the wide frequency range with a significant accuracy. The empty capacitance of this cell is so large about $200 \mathrm{pF}$. Detailed explanation of this cell has already been described elsewhere [32]. Cell A was also used to evaluate the precise value of the static dielectric constant. From $1 \mathrm{MHz}$ to $500 \mathrm{MHz}$ and from $100 \mathrm{MHz}$ to $10 \mathrm{GHz}, \mathrm{S}$-parameter method was employed, using two network analyzers (HP 4195A and HP 8510B). In the case of 4195A, a sample cell with the same structure as that of cell B was employed with empty capacitance of about $25 \mathrm{pF}$. In the case of 8510B, a parallel-plate-type cell with APC-7 connector (cell $C$ ) was used. More detailed information of this measurement has already been published [33]. To remove the effect of the stray capacitance and the error of geometric capacitance, some corrections were made to evaluate the complex permittivity.

Temperature of the dielectric cells was controlled using the temperature chamber (ESPEC MC811T) within the error $\pm 0.1 \mathrm{~K}$. Dielectric measurements were carried out at an interval of $10 \mathrm{~K}$ from 193 to $363 \mathrm{~K}$. In this study, glass transition temperature $\left(T_{g}\right)$ is defined as the temperature at which the peak frequency of the $\alpha$ relaxation is $10 \mathrm{mHz}$.

\section{RESULT AND DISCUSSION}

Figures 3 and 4 show the observed permittivity and dielectric loss of 1,2,6-hexanetriol. Data for every $10 \mathrm{~K}$ interval in the range of 193 to $363 \mathrm{~K}$ are indicated. The loci of dielectric loss show the main relaxation process ( $\alpha$ process) and the secondary relaxation process of the excess wing type. 


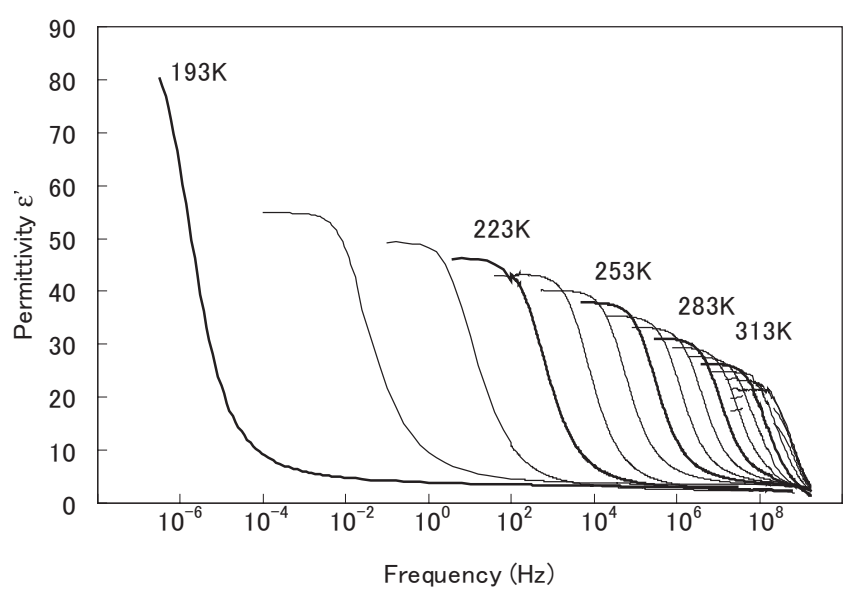

FIG. 3. Permittivity, $\varepsilon^{\prime}$, of 1,2,6-hexanetriol. The curves at every $10 \mathrm{~K}$ from 193 to $363 \mathrm{~K}$ are indicated.

The behavior of the dielectric loss such as no apparent secondary relaxation peak is similar to that of glycerol. This situation is quite different from that of sorbitol where clear loss peak is observed on the higher frequency side of the $\alpha$ process. To make quantitative analysis, we fitted the complex permittivity data by summing up the Davidson-Cole function [34] for the $\alpha$ process and the Cole-Cole function [35] for the secondary process. Thus, experimental results are well described using these functions. However, we are not going to discuss on the secondary process because the determination of dielectric parameters on this process contains significant ambiguity.

Figure 5 shows the Arrhenius plot for peak frequency of the $\alpha$ process for hexanetriol together with those for glycerol, threitol, xylitol, and sorbitol $[12,13]$. With decreasing temperature toward $T_{g}$, the relaxation frequency decreases drastically as seen in many glass formers. The loci of hexanetriol are close to that of glycerol, which show slower curvature and hence smaller fragility and $T_{g}$ than those of other sugar alcohols. This suggests that $N_{O H}$ is a more dominant factor than $N_{C}$ in describing the temperature dependence of $\alpha$ relaxation time of polyhydric alcohols.

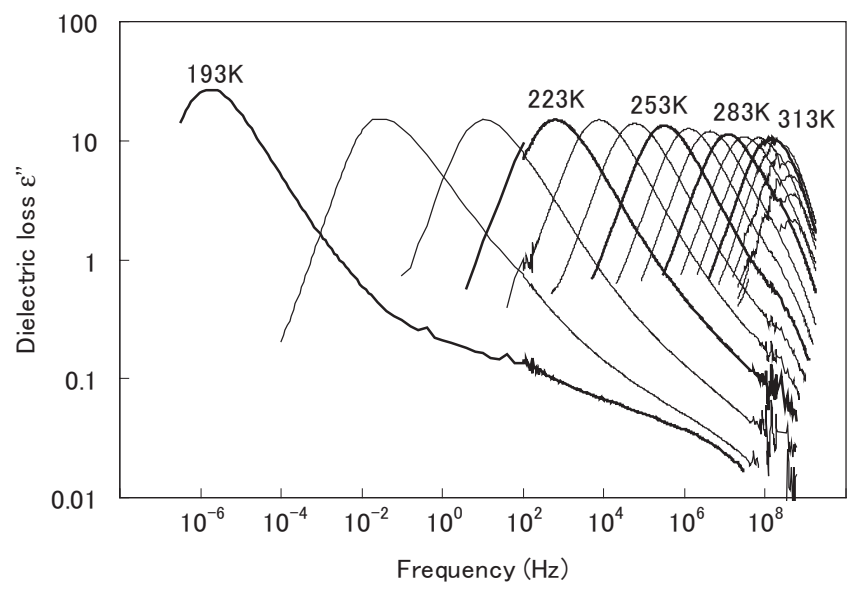

FIG. 4. Dielectric loss, $\varepsilon^{\prime \prime}$, of 1,2,6-hexanetriol. The curves at every $10 \mathrm{~K}$ from $193 \mathrm{~K}$ to $363 \mathrm{~K}$ are indicated. The loss spectrum exhibits main dispersion and excess wing. Such shape of spectrum is similar to that of glycerol rather than sorbitol (see also Fig. 2).

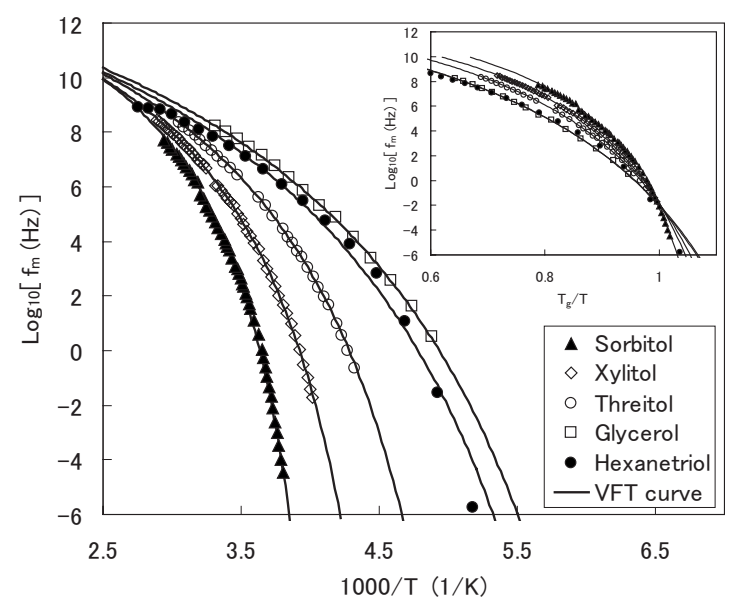

FIG. 5. Arrhenius plot for the peak frequency of main dispersion, $f_{m}$. Solid curves indicate the result of least square fitting with VFT equation. The curve for hexanetriol is close to that of glycerol. In the inset, $x$-axis is shown in reciprocal temperature normalized by glass transition temperature.

To discuss more quantitatively, the least-squares fitting with VFT equation [15-17] (solid curves) was performed against the peak frequencies measured at various temperatures. The VFT equation is explained by

$$
f_{m}=f_{0} \exp \left[-\frac{B}{T-T_{0}}\right],
$$

where $f_{m}$ is the peak frequency of $\alpha$ relaxation and $T$ temperature. Parameters $f_{0}, B$ and the Vogel temperature $T_{0}$ are fitting parameters. Since the choice of the $f_{0}$ value only gives rise to a small effect on the estimation of $T_{g}$ and fragility index, $f_{0}$ is set to be $10^{14} \mathrm{~Hz}$, which has been used in many previous studies $[12,13,36]$. The obtained fitting parameters were $B=2293 \mathrm{~K}$ and $T_{0}=137.5 \mathrm{~K}$. The fragility, $m$, and $T_{g}$ are calculated from obtained VFT parameters by using the following equations:

$$
\begin{aligned}
m= & -\left.\frac{d\left(\log _{10} f_{m}\right)}{d\left(T_{g} / T\right)}\right|_{T=T_{g}} \\
= & \frac{T_{0}}{B \log _{10} e}\left[\log _{10} f_{0}-\log _{10} f_{m}\left(T=T_{g}\right)\right]^{2} \\
& +\left[\log _{10} f_{0}-\log _{10} f_{m}\left(T=T_{g}\right)\right], \\
& T_{g}=T_{0}+\frac{B \log _{10} e}{\log _{10} f_{0}-\log _{10} f_{m}\left(T=T_{g}\right)},
\end{aligned}
$$

where $T_{g}$ is defined as the temperature where $f_{m}=10^{-2} \mathrm{~Hz}$. According to the fitting parameters obtained from the analysis described above, the values of $m$ and $T_{g}$ were determined as $51 \pm 4$ and $200 \pm 2 \mathrm{~K}$, respectively. The $T_{g}$ value is close to $206.4 \mathrm{~K}$ estimated using differential thermal analysis [37]. On the other hand, the present value of $m$ is not in good agreement with 74 calculated from a light-scattering data [37] in the literature [36]. In determination of the value of fragility we always have some experimental ambiguities due to limited frequency range, estimation of the value of $f_{0}$ and 


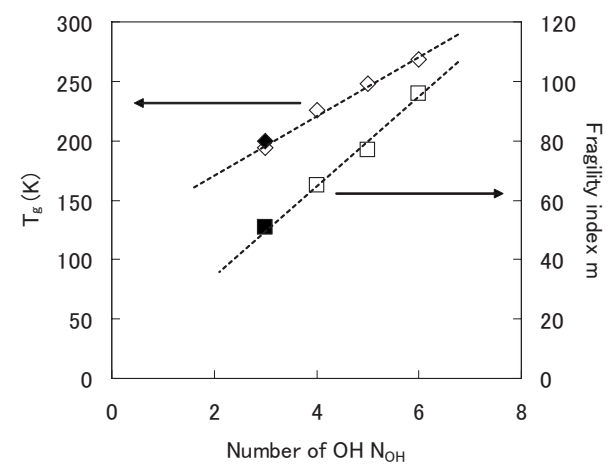

FIG. 6. Plots of glass transition temperature $\left(T_{g}\right)$ and fragility index $(m)$, against number of $\mathrm{OH}$ groups per a molecule $\left(N_{\mathrm{OH}}\right)$. Values for glycerol $\left(N_{O H}=3\right)$, threitol $\left(N_{O H}=4\right)$, xylitol $\left(N_{O H}=5\right)$ and sorbitol $\left(N_{O H}=6\right)$ are represented by open symbols and these for 1,2,6-hexanetriol $\left(N_{O H}=3\right)$ are shown by solid symbols. Diamonds and squares correspond to $T_{g}$ and $m$, respectively. Both of them exhibit systematic variation against $N_{\mathrm{OH}}$. Dashed line is depicted just for guide eyes.

so on. In the literature [37], VFT equation was evaluated by various experimental methods, light-scattering, dielectric relaxation and viscosity. Actually, fragilities calculated from these VFT equations distribute from 53 to 74 . Based on this distribution, the difference in $m$ between present work and some other works is considered not to be significant. In the present paper, all of the data including sugar alcohols are analyzed based on the common method in order to make a consistent comparison.

Figure 6 shows $m$ and $T_{g}$ against $N_{O H}$ for the data obtained in this study together with the data reported for sugar alcohols [13]. It is clear that relations of $m$ and $T_{g}$ to $N_{O H}$ for hexanetriol are similar to those for glycerol rather than for sorbitol. Moreover, linear dependence is revealed between the fragility index $m$ and $N_{O H}$. This result clearly suggests that the parameter $N_{O H}$ is more dominant to describe the $\alpha$ relaxation process compared to the parameter $N_{C}$. It is surprising that size of their molecules only gives rise to a small effect on the glass transition behavior.

To consider possible origin of the systematic trend of $m$ and $T_{g}$ with $N_{O H}$, the structure of hydrogen-bonding network was considered by analyzing the static dielectric constant. Since carbon chain is not dielectrically active, dielectric spectroscopy probes only the $\mathrm{OH}$ group. The effect of carbon chain gives rise to the further interaction between $\mathrm{OH}$ groups.

Temperature dependence of the static dielectric constant, $\varepsilon_{s}$, for hexanetriol and for the sugar alcohols [12] is shown in Fig. 7. In the case of sugar alcohols, static dielectric constants are almost the same. This can be understood in such a way that the number density of $\mathrm{OH}$ group is nearly equivalent in all the sugar alcohols. On the other hand, the static dielectric constant of hexanetriol is somewhat smaller than that of sugar alcohols. It is natural because the number density of $\mathrm{OH}$ group must be smaller than that of sugar alcohols. The dielectric constant of hexanetriol increases with decreasing temperature. This increase is steeper compared to the increase in linear temperature dependence. This temperature dependence can be reproduced using Curie-Weiss law as re-

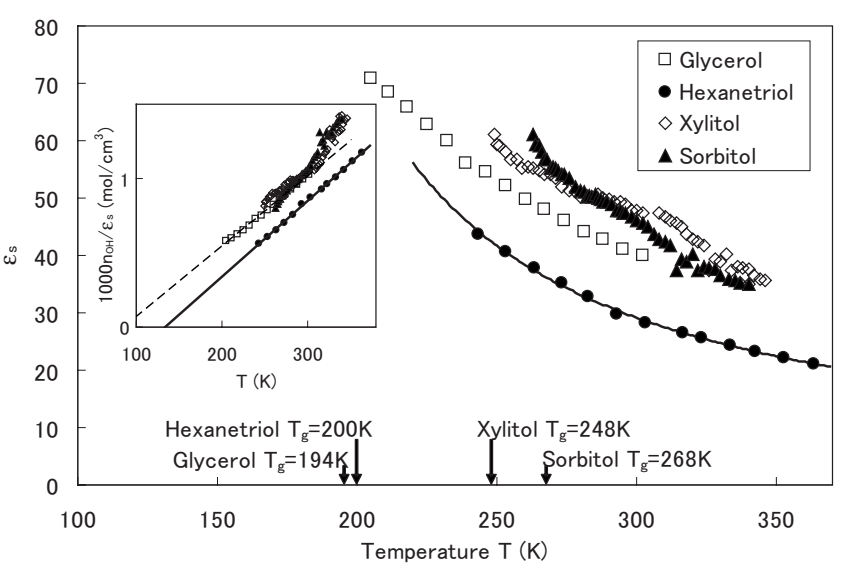

FIG. 7. Plots of static dielectric constant, $\varepsilon_{s}$, against temperature. The loci of all materials exhibit increase toward low temperature. Solid curve shows the result for least square fitting with the Curie-Weiss equation. The inset shows Curie-Weiss plot for sugar alcohols and hexanetriol. Reciprocal $\varepsilon_{S}$ normalized by number density of $\mathrm{OH}$ group, $n_{\mathrm{OH}}$, is plotted against temperature. The result for the fitting is also shown in this inset as a solid line. The dashed line is drawn just for guide eyes.

ported in sugar alcohols [7]. In the inset of Fig. 7, reciprocal dielectric constant is plotted against temperature (CurieWeiss plot). The dielectric constants, here, are normalized by number density of $\mathrm{OH}$ group, $n_{\mathrm{OH}}$. (Values of the density used in this plots are going to be described later.) In the Curie-Weiss plot, Curie-Weiss temperature dependence is represented as linear function and $x$ intercept corresponds to Curie temperature, $T_{C}$. The reciprocal dielectric constant for hexanetriol can be well described as a linear function as shown in solid line. Solid curve in the main part of Fig. 7 indicates the result of the least square fitting with the equation, $\varepsilon_{s}=4849 /(T-133.5)$. Value of obtained Curie temperature, $T_{C}=133.5 \mathrm{~K}$, is close to the Vogel temperature, $T_{0}$ $=137.5 \mathrm{~K}$. However it should be noted that since experimentally accessible dielectric constant is only within the temperature region far above $T_{C}$, the expected error based on rough estimation is considered to be very large which is about $\pm 30 \mathrm{~K}$. In the case of glycerol, similar Curie-Weiss temperature dependence has been reported by Schneider et al. [7]. (They reported that $T_{C}=100 \mathrm{~K}$, whereas $T_{0}=131 \mathrm{~K}$, which do not coincide with each other). Such Curie-Weiss temperature dependence in dielectric constant implies increasing of the association between neighboring $\mathrm{OH}$ groups.

Precise consideration for static dielectric constant can be obtained by using the theory of Kirkwood and Frölich [38]. They derived the following equations:

$$
\begin{gathered}
\varepsilon_{s}-\varepsilon_{\infty}=\frac{3 \varepsilon_{s}}{2 \varepsilon_{s}+\varepsilon_{\infty}}\left(\frac{\varepsilon_{\infty}+2}{3}\right)^{2} \frac{n g \mu_{0}^{2}}{3 \varepsilon_{0} k_{B} T}, \\
g=(1+z\langle\cos \theta\rangle),
\end{gathered}
$$

where $\mu_{0}$ is the isolated dipole moment, $\varepsilon_{s}$ is the static dielectric constant, $\varepsilon_{\infty}$ is the high-frequency limit of dielectric constant, $n$ is the number density of dipole moment, $\varepsilon_{0}$ is dielectric constant of vacuum, and $k_{B}$ is Boltzmann constant. 
Factor $g$ is called the Kirkwood correlation factor that exhibits an orientational correlation between considered dipole moment and its $z$ number of neighboring dipole. When $g$ $>1$, dipoles prefer to orient in a parallel direction while when $g<1$, they orient in an antiparallel direction. Usually, a dipole moment within the whole molecule is chosen as one dipole moment unit, $\mu_{0}$, which can be evaluated from dielectric constant in gas phase. Such treatment, however, can be valid only in a molecule without internal degrees of freedom. On the other hand, in the case of complex molecules such as polyhydric alcohols, there are strong intermolecular interactions and several degrees of freedom on the $\mathrm{OH}$ group orientation. Therefore, dipole moment of a whole molecule in liquid phase has possibly been changed into different values and directions from that in gas phase. Chelli et al. pointed out that abundance of each chain conformation in liquid phase is different from that in gas phase based on a molecular dynamic simulation of glycerol [26,27]. Therefore, it is natural to consider that the preferred orientation of each $\mathrm{OH}$ group is different in each phase. In a gas phase, the conformation of a carbon chain and the orientation of $\mathrm{OH}$ group are determined only by intramolecular interaction since intermolecular interaction is negligible. In a liquid phase, on the other hand, the conformations are affected by intermolecular interaction, such as hydrogen bonding. From these considerations, when the Kirkwood-Frölich theory is applied to polyhydric alcohols, it is not valid to use dipole moment of a molecule in gas phase as the parameter $\mu_{0}$ in Eq. (4). We therefore regarded dipole moment of $\mathrm{OH}$ group as $\mu_{0}$, instead that of the whole molecule. In this case, the parameter $g$ represents the $\mathrm{OH}-\mathrm{OH}$ orientational correlation and not molecular orientational correlation. In the system of hydrogen-bonding liquid, $\mathrm{OH}$ groups form hydrogenbonding network [25-29]. Since hydrogen bonding is a direction-specific interaction, formation of hydrogen bonding improves $\mathrm{OH}-\mathrm{OH}$ orientational correlation. For example, in the literature [38], tetrahedral hydrogen-bonding structure of water consisting of the nearest neighbors lead to $g \sim 2.33$, and this gives to the dielectric constant about 66 at $293 \mathrm{~K}$, which is roughly consistent with the experimental results. From this aspect, the orientational correlation factor $g$ can be interpreted as a parameter indicating hydrogen-bonding formation.

Figure 8 shows temperature dependence of orientational correlation factor $g$ regarding $\mathrm{OH}$ groups. The value $1.69 \mathrm{D}$ was used for the $\mathrm{OH}$ group dipole moment [39]. High frequency limit of dielectric constant $\varepsilon_{\infty}$ is obtained by taking the square of refractive index. Refractive index for glycerol and hexanetriol are 1.4746 [40] and 1.58 [40] at $293 \mathrm{~K}$ and wavelength $589 \mathrm{~nm}$, respectively. It should be noted that, since sorbitol and xylitol are crystal in ambient temperature, data for supercooled liquid are not available. Therefore, the refractive index of glycerol is used, instead of that of xylitol and sorbitol. Values of density used for glycerol, hexanetriol, xylitol, and sorbitol were $1.2613 \mathrm{~g} / \mathrm{cm}^{3}$ [40], $1.1049 \mathrm{~g} / \mathrm{cm}^{3}$ [40], $1.52 \mathrm{~g} / \mathrm{cm}^{3}$ [41], and $1.489 \mathrm{~g} / \mathrm{cm}^{3}$ [40], respectively. All of them are values obtained at $293 \mathrm{~K}$, where the values for sorbitol and xylitol are in the crystalline states. Although only the values taken at $293 \mathrm{~K}$ are used for this calculation, the effect leads only to a small error. Thermal expansion

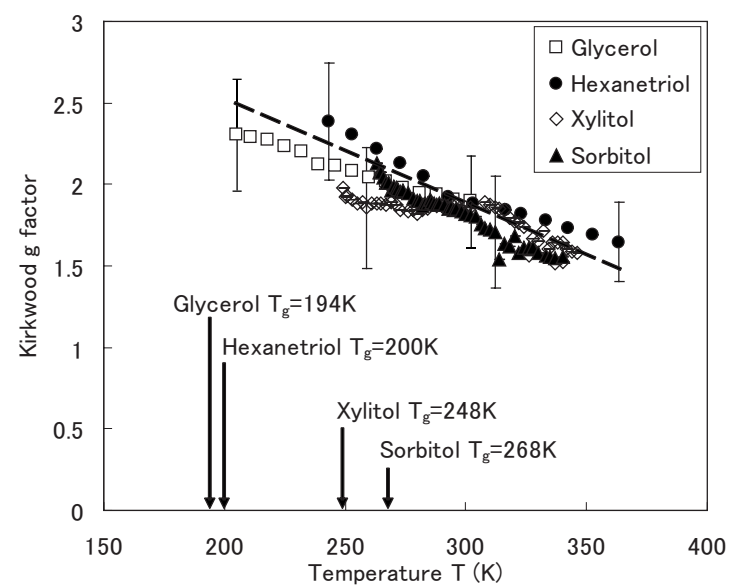

FIG. 8. Plots of orientational correlation factor $g$ regarding $\mathrm{OH}$ group against temperature, which is calculated from static dielectric constant in Fig. 7. All of them tend to increase with decreasing temperature and all loci give similar behavior. Dashed line is drawn just for guide eyes.

coefficient of glycerol is $4.90 \times 10^{-4} \mathrm{~K}^{-1}$ [39]. Temperature difference at lowest temperature reaches about $100 \mathrm{~K}$, and the possible error of $g$ is about $5 \%$. For sorbitol and xylitol, using the density in crystalline state instead of that in liquid state may also lead to a small error. Blazhnov et al. reported that difference of density between crystalline and liquid glycerol is about $0.1 \mathrm{~g} / \mathrm{cm}^{3}$ [42]. It follows that the error of $g$ is about $8 \%$. Error bars shown in Fig. 8 represent the cumulative error of $g$ estimated in such a way just described above.

For all the samples, the correlation factor $g$ exhibits slow increase with decreasing temperature, and surprisingly all loci follow a common behavior within the error. This common temperature dependence suggests that the inter-OH group structure is very similar among all of the polyhydric alcohols studied in this work. This also implies that the formation of hydrogen bonding with decreasing temperature is similar among polyhydric alcohols. Such a common structure is a possible origin of the systematic properties against $N_{O H}$ already discussed above. The increase in probability of forming hydrogen bond has also been reported by molecular dynamic simulation [27]. This is consistent qualitatively with the result of the present study.

From the systematic dielectric properties on the basis of $N_{O H}$ for all the polyhydric alcohols, we would like to propose a simple view on the molecular dynamics of supercooled polyhydric alcohols. A polyhydric alcohol molecule is restricted from the surrounding molecules by the $\mathrm{OH}$ group through hydrogen bonding. The probability of forming hydrogen bond increases with decreasing temperature and this is likely common among polyhydric alcohols. On the other hand, each $N_{O H}$ is different for each material and therefore the molecule with higher $N_{\mathrm{OH}}$ exhibits higher probability of connection with the neighbor molecules. In the view of the AG theory, these connected molecules are regarded as a cooperative rearranging region (CRR), which relaxes simultaneously and cooperatively. Since higher $N_{O H}$ results in the higher probability of connection, higher $N_{O H}$ gives rise to 
larger CRR. The difference in the size of CRR can results in the systematic $N_{O H}$ dependence of polyhydric alcohols.

\section{CONCLUSION}

The dielectric spectra of hexanetriol were obtained in a broadband frequency range of $1 \mu \mathrm{Hz}-10 \mathrm{GHz}$ and wide temperature range of 193-363 K. Obtained spectra were analyzed not only from the dynamics but also from the statics. In the former, systematic $N_{O H}$ dependence of $m$ and $T_{g}$ is revealed. This result supports that the dominant parameter for systematic character of sugar alcohols is $N_{O H}$ rather than $N_{C}$. In the latter, on the other hand, temperature dependence of the orientational correlation factor for $\mathrm{OH}$ groups are quite similar and they increase with decreasing temperature in all examined polyhydric alcohols. It is suggested from these results that the inter-OH group structure is similar among poly- hydric alcohols and the probability of hydrogen bonding increases with decreasing temperature. Although the inter-OH structure may be common in polyhydric alcohols, each $\mathrm{OH}$ group is associated by carbon chain. Therefore, the number of connected molecules reflects the number of $\mathrm{OH}$ groups per molecule, $N_{O H}$. This picture suggests a possibility that supercooled hydrogen-bonding liquid can be treated as a reduced model consisting of particles with $N_{O H}$ connecting sites. For future works, a variety of polyhydric alcohols has to be examined to test the generality of the results presented here.

\section{ACKNOWLEDGMENTS}

This work was partly supported by KAKENHI Grant No. 19340116, Grant-in-Aid for Scientific Research (B), from The Ministry of Education, Culture, Sports, Science and Technology (MEXT) of Japan.
[1] C. A. Angell, J. Non-Cryst. Solids 131-133, 13 (1991).

[2] G. E. Gibson and W. F. Giauque, J. Am. Chem. Soc. 45, 93 (1923).

[3] N. O. Birge and S. R. Nagel, Phys. Rev. Lett. 54, 2674 (1985).

[4] Y. H. Jeong, S. R. Nagel, and S. Bhattacharya, Phys. Rev. A 34, 602 (1986).

[5] E. Rössler, A. P. Sokolov, A. Kisliuk, and D. Quitmann, Phys. Rev. B 49, 14967 (1994).

[6] J. Wuttke, W. Petry, and S. Pouget, J. Chem. Phys. 105, 5177 (1996).

[7] U. Schneider, P. Lunkenheimer, R. Brand, and A. Loidl, J. Non-Cryst. Solids 235-237, 173 (1998).

[8] R. Böhmer and G. Hinze, J. Chem. Phys. 109, 241 (1998).

[9] K. Schröter and E. Donth, J. Chem. Phys. 113, 9101 (2000).

[10] A. Döß, M. Paluch, H. Sillescu, and G. Hinze, Phys. Rev. Lett. 88, 095701 (2002).

[11] A. Döß, M. Paluch, H. Sillescu, and G. Hinze, J. Chem. Phys. 117, 6582 (2002).

[12] A. Minoguchi, K. Kitai, and R. Nozaki, Phys. Rev. E 68, 031501 (2003).

[13] A. Minoguchi, T. Kaneko, H. Sotokawa, and R. Nozaki, J. Non-Cryst. Solids 352, 4742 (2006).

[14] In the present paper, dielectric permittivity, dielectric loss and dielectric constant mean relative one which is the permittivity normalized by dielectric constant of vacuum, $\varepsilon_{0}$ $=8.854 \mathrm{pF} / \mathrm{m}$.

[15] H. Vogel, Phys. Z. 22, 645 (1921).

[16] G. S. Fulcher, J. Am. Ceram. Soc. 8, 339 (1925).

[17] G. Tammann and W. Z. Hesse, Z. Anorg. Allg. Chem. 156, 245 (1926)

[18] H. Cohen and D. Turnbull, J. Chem. Phys. 31, 1164 (1959).

[19] D. Turnbull and H. Choen, J. Chem. Phys. 34, 120 (1961).

[20] G. Adam and J. H. Gibbs, J. Chem. Phys. 43, 139 (1965).

[21] W. Götze, Complex Dynamics of Glass-Forming Liquids (Oxford University Press, New York, 2009).

[22] K. L. Ngai, J. Phys.: Condens. Matter 12, 6437 (2000).
[23] K. L. Ngai, J. Phys.: Condens. Matter 15, S1107 (2003).

[24] P. G. Debenedetti and F. H. Stillinger, Nature (London) 410, 259 (2001)

[25] I. Ohmine, J. Phys. Chem. 99, 6767 (1995).

[26] R. Chelli, P. Procacci, G. Cardini, R. G. D. Valle, and S. Califano, Phys. Chem. Chem. Phys. 1, 871 (1999).

[27] R. Chelli, P. Procacci, G. Cardini, and S. Califano, Phys. Chem. Chem. Phys. 1, 879 (1999).

[28] U. Kaatze, R. Behrends, and R. Pottel, J. Non-Cryst. Solids 305, 19 (2002).

[29] Y. Yomogida and R. Nozaki, AIP Conf. Proc. 982, 350 (2008).

[30] R. Nozaki and S. Mashimo, J. Chem. Phys. 87, 2271 (1987).

[31] N. G. McCrum, B. E. Read, and G. Williams, Anelastic and Dielectric Effects in Polymeric Solids (Wiley, New York, 1967).

[32] M. Nakanishi and R. Nozaki, J. Non-Cryst. Solids (to be published).

[33] K. Murata, A. Hanawa, and R. Nozaki, J. Appl. Phys. 98, 084107 (2005).

[34] D. W. Davidson and R. H. Cole, J. Chem. Phys. 19, 1484 (1951).

[35] K. S. Cole and R. H. Cole, J. Chem. Phys. 9, 341 (1941).

[36] R. Böhmer, K. L. Ngai, C. A. Angell, and D. J. Plazek, J. Chem. Phys. 99, 4201 (1993).

[37] Th. Dorfmüller, H. Dux, G. Fytas, and W. Mersch, J. Chem. Phys. 71, 366 (1979).

[38] H. Fröhlich, Theory of Dielectrics (Oxford University Press, London, 1958).

[39] Handbook of Chemistry: Pure Chemistry, 5th ed., edited by Y. Iwasawa et al. (Maruzen Co., Ltd., Tokyo, 2004).

[40] CRC Handbook of Chemistry and Physics, 86th ed., edited by D. R. Lide et al. (Taylor \& Francis, London, 2004).

[41] The Merck Index, 14th ed., edited by M. J. O'Neil et al. (Merck \& Co. Inc., New Jersey, 2006).

[42] I. V. Blazhnov, N. P. Malomuzh, and S. V. Lishchuk, J. Chem. Phys. 121, 6435 (2004). 